\title{
II.
}

\section{Bericht über die vom October 1880 bis October 1882 behandelten Ohrenkranken.}

\author{
Yon
}

\author{
Dr. A. Marian \\ in Aussig (Böhmen).
}

In dem bezeichneten Zeitraume kamen zur Behandlung, resp. Untersuchung 540 Kranke (einschliesslich der 27 seit dem letzten Berichte $\left.{ }^{1}\right)$ Verbliebenen). Davon waren:

Männlichen Geschlechts 326 oder 60,3 Proc.

Weiblichen Geschlechts $214=39,7=$

Kinder (bis 15 Jahre) $154=28,5 \Rightarrow$

Erwachsene . . . $386=71,5=$

Dem Alter nach vertheilten sich die 540 Falle folgendermaassen:

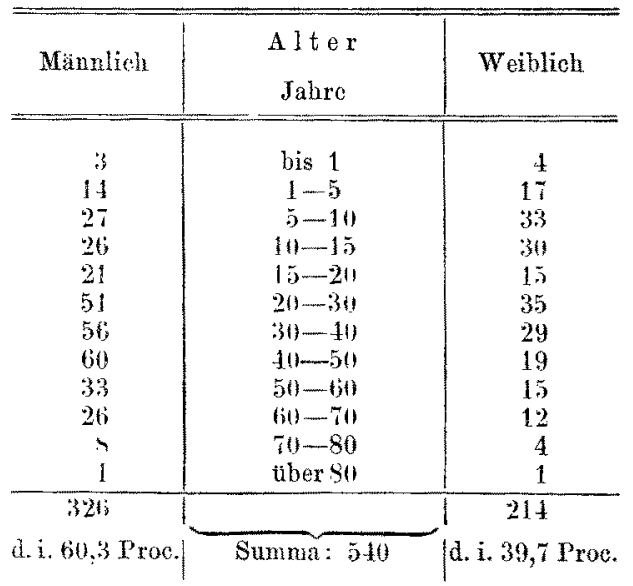

Von diesen wurden geheilt . . . . . . . . . $252=46,7$ Proc.

Wesentlich gebessert . . . . . . . . . . . . . $136=25,2=$

Blieben ungeheilt . . . . . . . . . $34=6,3=$

Ohne Behandlung ausgeblieben . . . . . . . $81=15,0=$

Erfolg der eingeleiteten Behandlung unbekannt : $: \quad 17=3,1=$

Gestorben . . . . . . . . . . . . . . $1=0,2=$

In Behandlung verblieben . . . . . . . . $19=3,5=$

1) s. dieses Archiv xVII. Bd. 
Nach Abzug der in Behandlung verbliebenen und der nicht in Behandlung genommenen Kranken ergibt sich für die geheilten Fälle 57,3 Proc., für die geheilten und gebesserten Fälle zusammen 88,0 Proc.

Die 540 Fälle zeigten 648 verschiedene Krankheitsformen, welche sich in folgender Weise vertheilten:

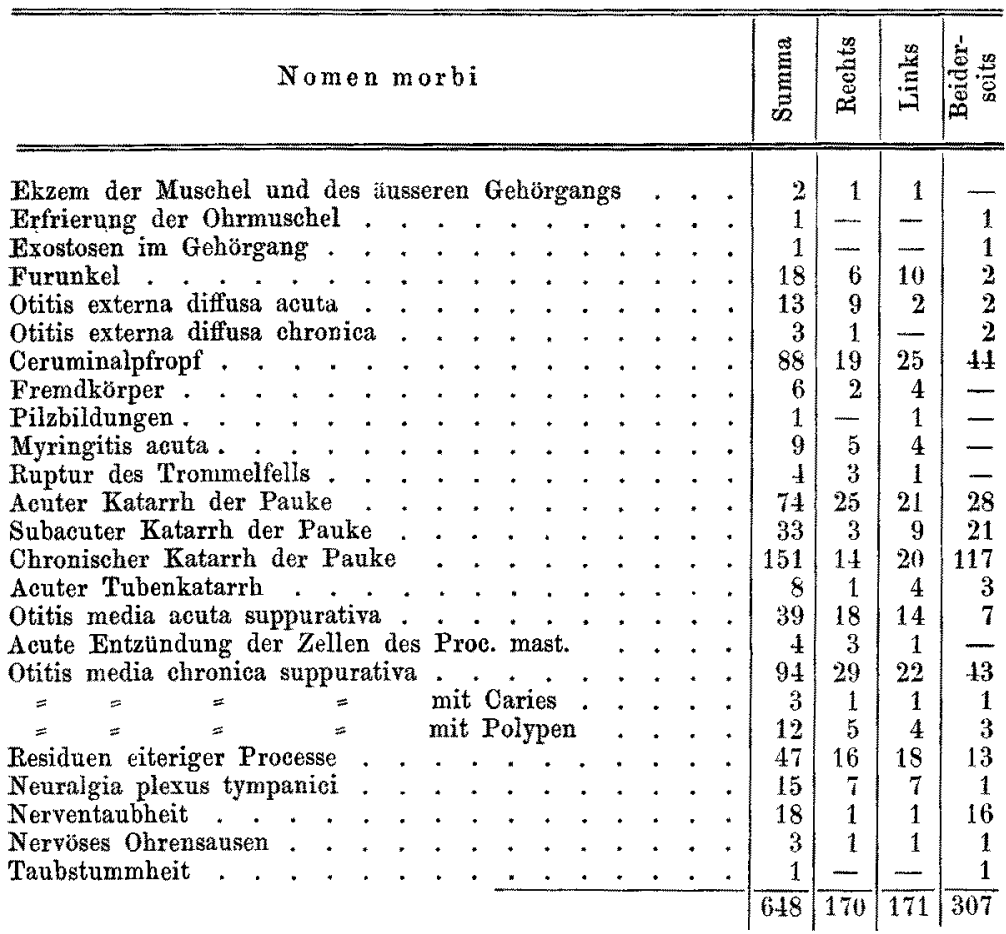

Bei diesen Fällen kamen gleichzeitig zur Behandlung:

Acuter Nasenkatarrh . . . . . . . . . . . . . . 32

Chronischer Nasenkatarrh . . . . . . . . . . 51

Ozaena . . . . . . . . . . . . . . . . . . . 10

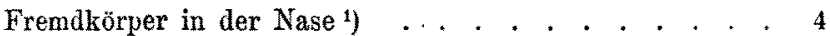

1) ohne gleichzeitige Erkrankung des Ohres, und zwar:

4jähr. Knabe: Bohne in der linken Nasenhöhle (Extraction mit der Pincette). 3 jähr. Knabe: Bohne in der linken Nasenhöhle (Extraction mit der gekrümmten Sonde).

4jähr. Knabe: Bohne in der rechten Nasenhöhle (Extraction mit der gekrümmten Sonde).

3 jähr. Mädchen: Glasperle in der rechten Nasenhöhle (Extraction mit der Pincette). 
Nasenpolypen . . . . . . . . . . . . . . . . . 7

Knochendefect in der Nasenhöhle . . . . . . . . . 1

Acuter Pharynxkatarrh . . . . . . . . . . . . . 11

Pharyngitis acuta cum tonsillitide . . . . . . . . . 5

Chronischer Pharynxkatarrh . . . . . . . . . . . 42

Pharyngitis granulosa (adenoide Vegetationen) . . . . . 33

Hypertrophie der Tonsillen . . . . . . . . . . . . . 28

Phlegmone des Halszellgewebes bei Angina tonsillaris . . 2

Ulcera und Cicatrices pharyngis specific. . . . . . . . 3

Diphtheritis der Nasen- und Pharynxschleimhaut . . . . 1

Von den 648 verschiedenen Krankheitsformen waren

doppelseitig $307=47,5$ Proc.

einseitig $\quad 341=52,5$

Von den letzteren betrafen das rechte Ohr 170, das linke 171.

Operationstabelle.

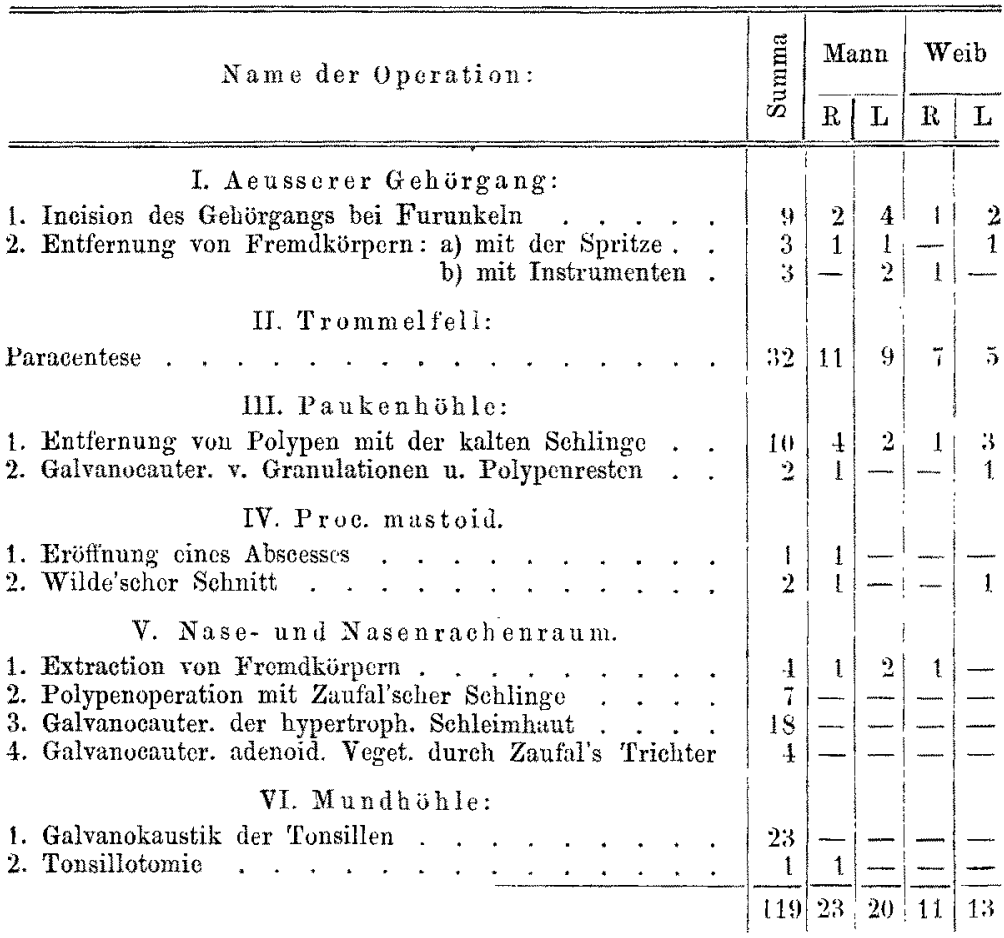

BEILAGE.

Ekzem der Muschel und des äusseren Gehörgangs:

$1 \mathrm{R}, 1 \mathrm{~L}$, geheilt.

18 monatl. Mädchen, Ekzem seit 6 Monaten bestehend, un- 
mittelbar nach einem Masernexanthem aufgetreten; Application von Pagenstecher'scher Salbe bringt die Affection binnen $12 \mathrm{Ta}$ gen zur Heilung.

6 jähr. Mädchen, Ekzem 5 Monate bestehend, nach Scarlatina und Diphtheritis mit Otitis media suppur. chron. desselben Ohres; Heilung nach 3 Wochen ebenfalls unter Anwendung von Pagenstecher'scher Salbe.

Congelatio conehae auris: $1 \mathrm{R}$ n. L, geheilt.

13 jähr. Kaufmannslehrling, vor 2 Jahren Erfrierung beider Obrmuscheln, seither starke Schwellung, blaurothe Verfärbung, sowie heftige brennende Schmerzen. Jodoformcollodiumbepinselung.

Exostosen im Gehörgang: $1 \mathrm{R} \mathrm{u}$. L, ohne Behandlung. 40 jähr. Beamte, im letzten Drittel des Gehörgangs bis nahe ans Trommelfell reichend, je von der vorderen und hinteren Wand ausgehend und das Lumen spaltförmig verengend.

Furunkel: R 6, L 10, R u. L 2, sämmtlich geheilt.

Alter: Von 10-20 Jabren 5, von 20-30 5, von 30-40 5, von $40-503$.

Beschäftigung: 4 Frauen (mit häuslicher Beschäftigung), 2 Kinder, 2 Bauern, je 1 Hafenmeister, Comptoirist, Student, Commis, Bremser, Rasirer, Schiffer, Schneider, Schuhmacher, Taglöhner.

Otitis externa diffusa acuta: $9 \mathrm{R}, 2 \mathrm{~L}, 2 \mathrm{R}$ u. L, sämmtlich geheilt.

Alter: Bis 10 Jahre 1, von 20-30 Jahren 3, von 30-40 2, von $40-505$, von $60-702$.

Beschäftigung: 6 Frauen, je 1 Kind, Lehrer, Beamter, Gensdarm, Gastwirth, Tischler, Siderolitharbeiter.

Otitis externa diffusa chronica: $1 \mathrm{R}, 2 \mathrm{Ru}$. L.

26 jähr. Kaufmann, 36 jähr. Eisenbahnconducteur, 74 jähr. Frau.

Ceruminalp fröpfe: $19 \mathrm{R}, 25 \mathrm{~L}, 44 \mathrm{R}$ u. $\mathrm{L}$.

Alter: Von 1-10 Jahren 9, von 10-20 5, von 20-30 6, von $30-4013$, von $40-5014$, von $50-6019$, von $60-7014$, von $70-808$.

Beschäftigung: 19 Bauern, 16 Frauen, 12 Kinder, 6 Tagarbeiter, 5 Beamte, 4 Kaufleute, 4 Gastwirthe, je 2 Geistliche, Schuhmacher, Maurer, je 1 Privatier, Fabrikant, Student, Getreidehändler, Viehhändler, Fleischbauer, Schmied, Tischler, Binder, Schiffer, Dachdecker, Schiffbauer, Fuhrmann, Glasarbeiter, Apothekerlaborant, Kanzleidiener. 
Fremdkörper: $2 \mathrm{R}, 4 \mathrm{~L}$.

In sämmtlichen Fällen waren keine Extractionsversuche vorausgegangen und gelang die Entfernung ohne jegliche Schwierigkeiten.

2jähr. Mädchen, Erbse im linken Gehörgang.

4 jähr. Knabe, Johannisbrodkern links.

12 jähr. Knabe, Gerstenkorn rechts. Sämmtlich dureh Ausspritzen entfernt.

38 jähr. Bauersfrau, seit Kindheit Glasperle im rechten Ohr. Dieselbe lag, ohne irgend welche Beschwerden zu verursachen, in etwas Cerumen eingebettet im mittleren Drittel des Gehörgangs und wurde mit der gekrümmten Sonde leicht entfernt.

29 jähr. Schiffer und 60 jähr. Specereiwaarenhändler, je einen bereits monatelang im $\mathrm{Ohr}$ befindlichen Baumwollpfropf.

Pilzbildung im Ohr: 1 L.

77 jähr. Häusler, Aspergillus flavesc. im linken $\mathrm{Ohr}$, starke Auflockerung des Trommelfells, Application von Acidum boricum in Pulverform mit gutem Erfolg.

Myringitis acuta: $5 \mathrm{R}, 4 \mathrm{~L}$.

Alter: Von 1-10 Jahren 1, von $20-303$, von $30-401$, von $40-503$, von $50-601$.

Beschäftigung: 2 Frauen mit häuslicher Beschäftigung, je 1 Kind, Bäuerin, Taglöhnerin, Schiffer, Schneider, Gastwirth, Arzt.

In 2 Fällen konnte als Ursache der Entzündung Eindringen kalten Wassers ins Ohr beim Baden constatirt werden.

Rupturdes Trommelfells: $3 \mathrm{R}, 1 \mathrm{~L}$.

69 jähr. Häusler, frische Ruptur im hinteren unteren Quadranten des atrophischen, im vorderen Quadranten ausgedehnte Verkalkung zeigenden Trommelfells in Folge mehrfacher Schläge aufs Ohr. Ruptur rechtwinklig mit einwärts gestiilpten, bis an das spatelförmige Ende des Hammergriffs heranreichenden Rändern und 2 streifenförmigen Blutextravasaten. Am 29. Tage nach der Verletzung gerichtliche Verhandlung, wonach die Verletzung: als schwere ohne bleibenden Nachtheil und ohne besondere Folgen definirt wurde.

18 jähr. Dienstmädchen, gleichfalls Ruptur in Folge einer Ohrfeige; ähnlicher Befund wie im vorigen Falle.

35 jähr. Frau hatte ror 6 Tagen mit einem Federhalter im rechten Ohr gebohrt, kam dabei mit dem rechten Arm an die Thür und stiess sich die Spitze des Federhalters tief ins Ohr; sofort heftiger Schmerz und etwas Blutnng. Spatelförmige Per- 
foration des sehr stark aufgelockerten Trommelfells, mässige Eiterung, bedeutende Verminderung der Hörweite; Application von Borsäure, Heilung nach 6 Wochen mit vollständig normaler Hörfähigkeit.

25jähr. Bauernknecht hatte sich vor 12 Tagen beim Aufladen eines Wagens einen Strohbalm ins linke Ohr gestossen. Sehr starke Schmerzen und Ohrenfluss, ovale Perforation im hinteren unteren Quadranten des sebr stark aufgelockerten Trommelfells; Borsäureapplication, Heilung nach 3 Wochen.

Acuter Katarrh der Paukenböhle: $25 \mathrm{R}, 21 \mathrm{~L}, 28 \mathrm{R}$ und $L$.

Alter: Von 1-10 Jahren 10, $10-2012,20-3015,30-40$ $13,40-5014,50-607,60-703$.

Beschäftigung: 19 Kinder, 8 Frauen, 6 Bauern, 5 Beamte, 5 Schiffer, 3 Commis, je 2 Fuhrleute, Viehhändler, Diurnisten und Kaufleute, je 1 Schuldirector, Lehrer, Musiklehrer, Geistlicher, Student, Bergwerksbesitzer, Conducteur, Maschinenfiuhrer, Weichenwärter, Gastwirth, Schuhmacher, Schneider, Maurer, Fleischhauer, Binder, Portier, Bildhaner, Steinmetz, Taglöhner und Taglöhnerin.

Behandlungsresultat: 3 ohne Behandlung, 58 geheilt, 10 gebessert, 1 ungeheilt, in 2 Fällen der Erfolg unbekannt.

Subacuter Katarrh der Pauke: $3 \mathrm{R}, 9 \mathrm{~L}, 21 \mathrm{R}$ u. L.

Alter: Von 1-10 Jahren 7, von 10-20 7, von $20-303$, von $30-407$, von $40-502$, von $50-605$, von $60-702$.

Beschäftigung: 13 Kinder, 5 Bauern, 4 Frauen, 2 Kaufleute, je 1 Arzt, Bergwerksbesitzer, Bergmann, Soldat, Spitzenhändler, Portier, Tischler, Schmied, Tagarbeiter.

Behandlungsresultat: 4 ohne Behandlung, 23 , geheilt, 6 gebessert.

Chronischer Katarrh der Pauke: $14 \mathrm{R}, 20 \mathrm{~L}, 117 \mathrm{R}$ und $\mathrm{L}$.

Alter: Von 1-10 Jahren 16, von 10-20 19, von 20-30 24, von $30-4020$, von $40-5026$, von $50-6022$, von $60-7018$, von $70-804$, über 802 .

Beschäftigtang: 33 Frauen, 28 Kinder, 19 Bauern, je 7 Kaufleute und Beamte, 6 Schiffer, 4 Tagarbeiter, 3 Studenten, je 2 Banmeister, Gutsverwalter, Geistliche, Lehrer, Commis, Schneider, Schmiede, Bäcker, Fabrikarbeiterinmen, je 1 Schiffbauer, Schlosser, Wachmann, Gastwirth, Postmeister, Schulinspector, Färber, Werkführer, Bahnarbeiter, Weichenwärter, Maurer, Schuhmacher, 
Uhrmacher, Müller, Steinmetzer, Fuhrmann, Bergmann, Seifensieder, Viehhändler, Eisenhändler, Kleiderhändler, Seiler, Glasarbeiter, Zimmermann, Branntweinschänker und Dienstmädchen.

Behandlungsresultat: 44 ohne Behandlung, 25 geheilt, 55 gebessert, 19 ungeheilt, bei 2 der Erfolg unbekannt; in Behandlung verblieben 6 .

Acuter Tubarkatarrh: $1 \mathrm{R}, 4 \mathrm{~L}, 3 \mathbf{R}$ u. L.

Alter: Von 10-20 Jahren 2, von $20-30$ 2, von $30-401$, von $40-502$, von $50-601$.

Beschäftigung: 2 Kinder, 2 Frauen, 2 Bergleute, je 1 Schneider und Todtengräber.

Behandlungsresultat: 6 geheilt, 1 gebessert, in 1 Fall Erfolg der Behandlung unbekannt.

Otitis media suppurativa acuta: $18 \mathrm{R}, 14 \mathrm{~L}, 7 \mathrm{R}$ u. L. Alter: Von 1-10 Jahren 13, von 10-20 5, von 20-30 6, von $30-402$, von $40-5011$, von $50-601$, von $60-701$.

Beschäftigung: 16 Kinder, 5 Frauen, 3 Beamte, je 2 Bauern und Tagarbeiter, je 1 Schuldirector, Gastwirth, Maschinenmeister, Comptoirist, Gensdarm, Locomotivheizer, Bremser, Steinmetzer, Maurer, Beekerlehrling, Knecht.

Behandlungsresultat: 25 geheilt, 6 gebessert, 2 mit unbekanntem Erfolg, 1 gestorben, 5 in Behandlung.

Der letal abgelaufene Fall betraf ein 4jähriges Mädchen, welches im Verlaufe von Scarlatina: Diphtheritis des Pharynx, der Tonsillen, der Nasenschleimhant und der linken Paukenhöhle bekam und am 25. Krankheitstage an Herzparalyse starb.

Während sonst bei den croupösen und diphtheritischen Rachenentzïndungen die Paukenböble nur von einer einfachen, nicht diphtheritischen Entzündung befallen wird (Sehwartze), bot sich hier das Bild einer der Continuität nach vom Plarynx nud der Nasenhöhle fortschleichenden diphtheritischen Paukenhöhlenentzindung dar. Als ich am 18. Tage der Entzundung das Kind das erste Mal untersuchte, fand ich bereits das Trommelfell nahezu vollständig zerstört, die Pankenhöhle sowie den Gehörgang mit diphtheritisehen Membranen durchsetzt, die sich äusserst schwer entfernen liessen und unter denen dam eine lebhaft granulirende, äusserst leicht blutende Geschwürsfläche zu 'Tage trat. Merkwürdig erscheint es, dass bei der grossen Intensität des diphtheritischen Processes namentlich anf der Nasensehleimbaut (beide Nasenhöhlen waren bei jedesmaliger Untersuchnng, trotzdem dic ärztlichen Anordnungen peinlichst genau befolgt wurden, mit 
äusserst übelriechendem eiterigen Secret angefüllt), sich die Entzündung nur auf e in Ohr fortpflanzte, während das andere (rechte) vollkommen intact blieb. Auch $\mathrm{Wreden}$, der das Vorkommen einer diphtheritischen Entzïndung des Mittelohres zuerst bei 18 Kindern im Gefolge von Scarlatina beobachtete, theilt mit, dass die Entzïndung sehr selten einseitig auftrete. Die therapeutischen Maassnahmen bestanden in diesem Falle in stiundlichen Ohr- und Nasenrachenausspritzungen mit Aqua calcis, dann 3 proc. Borsäurelösung, Bepinselungen der erkrankten Rachenpartien mit Carbolglycerin, innerlicher Darreichung von Chinin, Kali chloricum etc.; eine spontane Abstossung der diphtheritischen Membranen im $\mathrm{Ohr}$ konnte jedoch während der ganzen Behandlungsdauer (8 Tage) bis zum letalen Ende nicht beobachtet werden.

Acute Entzündung der Zellen des Warzenfortsatzes: $3 \mathrm{R}, 1 \mathrm{~L}$; alle geheilt.

Alter: 11, 21, 41, 51 Jahre.

Beschäftigung: 1 Knabe, 1 Mädchen, 1 Diurnist, 1 Bauer.

Otitis media suppurativa chronica: $29 \mathrm{R}, 22 \mathrm{~L}, 43$ $R$ und $L$.

Alter: Bis 1 Jahr 4, von 1-10 Jahren 34, von 10-20 24, von $20-3014$, von $30-409$, von $40-505$, von $50-602$, von $60-701$, von $70-801$.

Beschäftigung: 53 Kinder, 11 Franen, 6 Dienstmädchen, 3 Bauern, je 2 Eisenbahnbeamte, Kaufleute, Studenten, Schlosser, je 1 Telegraphenbeamter, Maschinenbauer, Viehbändler, Schiffer, Zinngiesser, Kellner, Mtiller, Schmied, Weber, Schuhmacher, Wirthschafterin, Hebamme, Taglöhnerin.

Als veranlassende Ursache konnte $23 \mathrm{mal}$ Scharlach, $5 \mathrm{mal}$ Masern, 6 mal Diphtheritis, 2 mal Variola, 12 mal Scrophulose, 4 mal Typhus, 2 mal chronischer Nasenkatarrh, 3 mal Keuchhusten, 1 mal Rhachitis, 3 mal kaltes Bad, 1 mal Trauma constatirt werden.

Behandlungsresultat: 17 geheilt, 48 gebessert, 17 ohne Behandlung, 3 mit unbekanntem Erfolg, 5 ungeheilt, 4 in Behandlung verblieben.

Unter den bei chronischen als auch acuten Mittelohreiterungen angewendeten Mitteln steht auch diesmal die Borsäure oben an und waren auch diesmal die Erfolge, die mit diesem Medicament erzielt wurden, fast durchgehends äusserst zufriedenstellende. 
Otitis media suppurativa chronica mit Caries des Felsenbeins: $1 \mathrm{R}, 1 \mathrm{~L}, 1 \mathrm{R}$ u. L.

11 jähr. Mädchen, seit $3 \frac{1 / 2}{2}$ Jahren beiderseitige Otitis med. supp. chron. post scarlatinam, Caries des linken Proc. mastoid. Nach Abstossung und Extraction mehrerer Sequester durch die erweiterte Fistel am Proc. mastoid. Heilung.

6 jähr. Knabe, seit 4 Jahren beiderseitige Otitis med. supp. chron. mit Caries des Felsenbeins. Extraction eines nekrotischen, der hinteren Paukenhöhlenwand angehörenden Knochens - Heilung - seit 2 Jahren kein Recidiv der chronischen Entzündung.

10 jähr. Mädchen, seit 6 Jahren beiderseitige Otorrhoe post scarlatinam, tiefe Knochennarben am Proc. mast. beiderseits, häufiges Abgehen von "Knochensand" mit dem Eiter, vollständiger Defect beider Trommelfelle, sämmtlicher Gehörknöchelchen, stark granulirende Paukenhöhlenschleimhaut.

Otitis media suppur. chron. mit Polypenbildung: $5 \mathrm{R}, 4 \mathrm{~L}, 3 \mathrm{R}$ u. L.

Alter: Von 1-10 Jahren 2, von 10-20 4, von 20-30 2, von $30-403$, von $50-601$.

Beschäftigung: 4 Kinder, je 1 Bergverwalter, Lehrer, Maschinenmeister, Weber, Schiffer, Lehrerin, Fabrikarbeiterin, Dienstmädchen.

Behandlungsresultat: 6 gebessert, 1 ungeheilt, 3 ohne Behandlung, 2 in Behandlung verblieben.

Residuen eiteriger $\operatorname{Pr} 0$ cesse: $16 \mathrm{R}, 18 \mathrm{~L}, 13 \mathrm{R}$ u. L.

Alter: Von 1-10 Jahren 7, von 10-20 12, von 20-30 7, von $30-408$, von $40-5011$, von $60-701$, von $70-801$.

Beschäftigung: 12 Kinder, 8 Bauern, 5 Frauen, 4 Dienstmädchen, 3 Taglöhnerinnen, je 2 Beamte, Gastwirthe, Bergarbeiter, je 1 Kaufmann, Student, Eisenbahnconducteur, Bäcker, Schneider, Binder, Weber, Schmied, Wirthschafterin.

Behandlungsresultat: 11 geheilt, 25 gebessert, 5 ohne Behandlung, 3 ungeheilt, 3 in Behandlung verblieben.

Neuralgia plexus tympanici: $7 \mathrm{R}, 7 \mathrm{~L}, 1 \mathrm{R}$ u. L.

Alter: Von 10-20 Jahren 4, von 20-30 8, von $30-402$, von $60-701$.

Beschäftigung: 2 Mädchen, 10 Frauen mit häuslicher Beschäftigung, 1 Dienstmädchen, 1 Fabrikarbeiterin, 1 Locomotivführer.

Behandlungsresultat: 11 geheilt, 1 gebessert, 3 mit unbekanntem Erfolg. 
Nerventaubheit: $1 \mathrm{R}, 1 \mathrm{~L}, 16 \mathrm{R}$ u. L.

Alter: Von 1-10 Jahren 4, von 10-20 2, von 20-30 2, von $30-405$, von $40-503$, von $50-601$, von $60-701$.

Beschäftigung: 6 Kinder, 4 Frauen, 2 Taglöhner, je 1 Modelleur, Buchbinder, Maschinenschlosser, Kaufmann, Händler (ausgedienter Artillerist), Fabrikarbeiterin.

Ursache: 4 mal Scharlach und Diphtheritis, 3 mal Meningitis, 2 mal Trauma (1 Fall auf den Kopf, 1 mal Sehuss), 1 mal Typhus, 1 mal Typhus und Scharlach, 1 mal Soldatendienst bei der Artillerie, $6 \mathrm{mal}$ war die veranlassende Ursache nicht $\mathrm{zu}$ eruiren.

Behandlungsresultat: 2 gebessert, 8 ungeheilt, 8 obne Behandlung.

Taubstummheit: $3^{1 / 2}$ jähr. Knabe, bei negativem Ohrbefund ohne vorausgegangene Krankheit.

Nervöses Ohrensausen (mit negativem Ohrbefund und intacter Hörfähigkeit): $1 \mathrm{R}, 1 \mathrm{~L}, 1 \mathrm{R}$ u. L.

Alter und Beschäftigung: 26 jähr. Schlosser, 42 jähr. Beamte, 60 jäbr. Frau.

Behandlungsresultat: 1 ohne Behandlung, 1 ungeheilt, 1 in Behandlung befindlich.

Veranlasst durch die Arbeiten von $\mathrm{B} \mathfrak{i} \mathrm{rkner}, \mathrm{M} 0$ os, Schwabach und Pollnow „über Ohrenkrankheiten bei Eisenbahnbediensteten " in diesem Archiv ${ }^{1}$ ) stellte ich gelegentlich der Ausarbeitung der Rubrik "Beschäftigung" die mir bisher zur Untersuchung und Behandlung vorgekommenen Fälle von Ohrenerkrankungen bei Eisenbahnbediensteten in nachstehender Tabelle (S. 23) zusammen.

Es finden sich in dieser Tabelle auch die Verkehrsbeamten aufgenommen, welche sowohl im Dienstbetriebe, namentlich auf grösseren Stationen mannigfache Signale zu beachten haben, als auch im Verkehr mit dem Publicum (z. B. beim Kassendienste) ein annähernd normales Gehör nicht werden entbehren können. Zum Beweis dessen mag die Bemerkung dienen, dass von den drei mit chronischem Paukenhöhlenkatarrh behafteten Beamten zwei vom Verkehrs- zum Kanzleidienste in den Directionsbureaus versetzt werden mussten, da sie ersterem wegen der immer bedeutender werdenden Verminderung ihrer Hörfähigkeit nicht mehr gerecht werden konnten.

1) s. IX. Bd., 4. Heft, XVII. Bd., 1. u. 2. Heft, XVIII. Bd., 1. u. 2. Heft. 


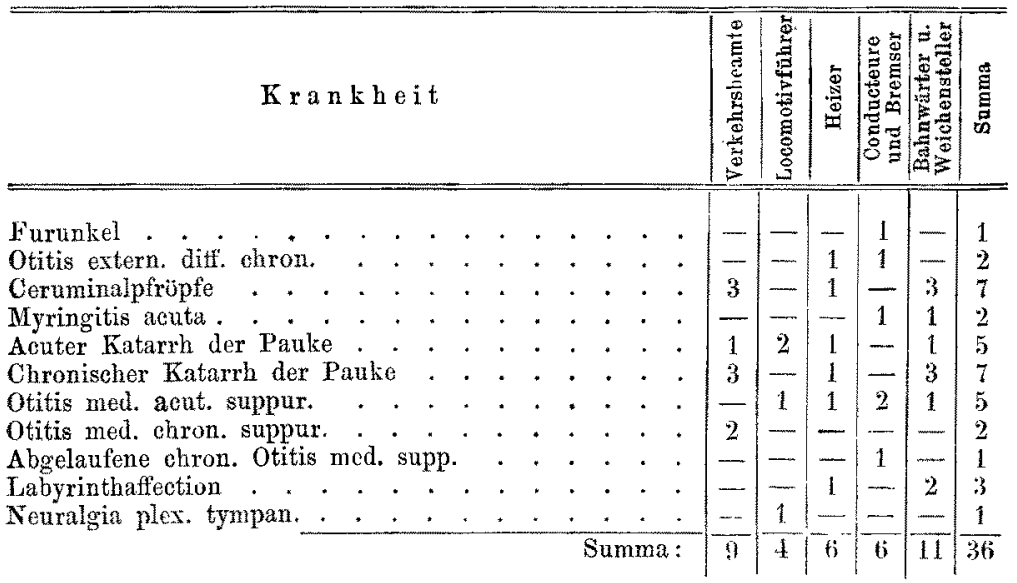

Man ersieht aus der Tabelle, dass die Eisenbabnbediensteten einen immerhin erbeblichen Procentsatz zu den von verschiedenartigen Erkrankungen des Gehörorgans Betroffenen stellen, und dass diesem Umstande wohl von den maassgebenden Kreisen Rechnung zu tragen sein wird, wenn im Interesse des Dienstes und der öffentlichen Sicherheit schwere Schäden und Unglücksfälle verhïtet werden sollen.

Ein Umstand mag hierbei noch hervorgehoben werden, der meiner subjectiven Erfahrung nach hier ins Gewicht fällt: Die Nothwendigkeit der Behandlung der Erkrankungen des Gehörorgans (namentlich acuter) durch einen mit Diagnose und Therapie von Ohrenkrankheiten vertrauten Arzt. Bereits mehr als einmal hatte ich Gelegenheit die Wahmehmung zu machen, dass Eisenbabnbedienstete mit acuter Mittelohrentzündung oder anderen acuten Erkrankungen, die bereits schon eine wesentliche Beeinträchtigung der Hörweite nach sich gezogen hatten, noch den Dienst als Locomotivführer, Heizer oder Weichenwärter versahen oder versehen mussten, da entweder von dem betreffenden Bahnarzte der schon immerhin bedenklichen Erkrankung nicht die gebührende Beachtung geschenkt, oder aber der Kranke schon in einem Stadium als dienstfähig bezeichnet wurde, wo der Krankheitsprocess noch keineswegs abgelaufen war.

Es lassen sich mithin dem von Moos angeregten Thema noch weitere Gesichtspunkte abgewinnen, und dürften obige Reflexionen vielleicht den Ausgangspunkt zu fortgesetzten Untersuchungen und Vorschlägen in dieser Frage bilden. 